\title{
THE SCOPE OF SYMPATHETIC DENERVATION IN POLIOMYELITIS
}

\author{
I. J. Zadikoff, Surgeon, Johannesburg
}

Poliomyelitis is a general infection caused by a filterable virus with its most marked effects on the nervous system. Pericellular and perivascular lymphocytic infiltration leads to destruction of nerve cells not only in the Central Nervous System but also in the Autonomic Nervous System. The effects on the Sympathetic Ganglia are always present to a greater or lesser extent, but these are often neglected in the storm of the general infection and motor nerve pathology.

The Sympathetic ganglia, like the anterior horn cells, are irregularly involved in this disease and thus the resultant vasospastic systemic symptoms in the acute stage of the disease are aggravated or lessened. Vasoconstrictor sequelae such as coldness, discolouration, sweating, pain, muscle spasm and occasionally alternating flushing and pallor are commonly found. In the late, heartbreaking stage of crippling paralysis, the affected limbs are commonly cold, woist and cyanotic. The diminished arterial supply in a paralytic limb, the bulk of which is poorly nourished fat, often produces, in cold weather, multiple reddish purple subcutaneous nodules (erythema induratum) which may ulcerate as a result of fat necrosis.

Associated with the paralysis due' to the involvement of the motor nerves, there is vasoconstriction in the muscles and thus diminished blood circulation. This aggravates the disuse atrophy due to the paralysis, pain, muscle spasm and in all probability, as Sister Kenny states, the atroplyy resulting from muscle incoordination and mental disorientation. The aggravating factor of a "cold" muscle is commonly noticed by parents who state that the limb is much more useful on waking in th morning, better in summer than in winter and poorer after a swim in a cold swimming bath. All athletes are aware of the fact that warm muscles act more efficiently than cold ones.

In time, bones too, particularly young growing ones, are affected as a result of the muscle inactivity and diminished blood circulation in the limb. The younger the patient and hence the more the bones are yet to grow, the greater the inactivity of the muscles as a result of paralysis, and the more marked the vasospasm, the more marked will be the ultimate shortening of the limb. Improvement of the circulation will thus diminish the detrimental effects of the muscle paralysis on the growth of bone.

Release of vasoconstriction is essential for the successful therapy in these cases. Vasospasm is not an unalterable pathological lesion but a physiological and functional derangement of the sympathetic nervous supply of the vessels, and is, for the present, one of the few controllable factors in the progress of the disease: In all stages of poliomyelitis whether in the acute or chronic stages, it merits treatment pari passu with the other pathological processes. All treatment for the lesion in the Autonomic Nervous System, whether conservative or radical is based on the release of this vasoconstriction. Heat applied locally to the acutely affected limb can be in the form of lot packs, fomentations, lamps, or reflexly by heating other parts of the body. This has to be repeated at frequent intervals. In the clironic stages, the wearing of long woollen stockings and gloves, and if possible living in a warm climate, particularly in winter, helps the cold limb.

Drugs, mainly acting by virtue of temporarily blocking the sympathetic ganglia have been found to be very useful particularly in the actue stage. Etamon, Priscol, Tolazoline and Cyclospasmol to mention only a few, have all been used with a varying amount of success in the acute stages but, infortunately to a much lesser extent, in my experience, in the convalescent and chronic stages of the disease. Direct injection of the sympathetic ganglia with local anaesthetics, has a temporary effect and rarely breaks the cycle permanently. It is painful, not entirely free from danger and unnecessary in the establishment of the diagnosis of vasospasm in this condition.

Radical treatment consists of operative preganglionic interruption of the sympathetic nerves to the affected limb. There is no associated arterial or venous disease other than the vasospasm and thus the post-operative release is immediate, maximal and usually permanent. Post-operatively the limb as a whole becomes more comfortable, skin lesions heal and in young patients bone growth improves. Subsequent orthopedic procedures, too, are commonly less extensive, necessary orthopedic splints are worn more comfortably, and the muscles become more responsive to the ministrations of the physiotherapist. 